\title{
Evaluación electroquímica de nanoestructuras Fe/MWCNT-Pt y Fe/MWCNT-Pt-Pd como materiales de cátodos multifuncionales con potencial aplicación en el mejoramiento de la calidad de agua tratada
}

\author{
Brenda Borbón Jara, ${ }^{*}$ Alejandro Medel, * Zaira Bedolla Valdez,* \\ Gabriel Alonso Núñez,* Mercedes T. Oropeza Guzmán*
}

\begin{abstract}
RESUMEN: En este artículo se presenta una estrategia de evaluación electroquímica para materiales nanoestructurados con potencial uso en cátodos multifuncionales, con el fin de incrementar la capacidad de degradación y eliminación de materia orgánica recalcitrante en agua tratada por vía electroquímica. El principal objeto de estudio es la producción de radical hidroxilo $\left({ }^{\circ} \mathrm{OH}\right)$, que es identificado como un agente altamente oxidante ( $2.8 \mathrm{~V}$ vs. ENH), únicamente superado por el flúor ( 3.03 v vs. ENH). Este radical suele formarse en la superficie de un ánodo catalítico o por reacciones tipo Fenton; sin embargo, en este artículo se demuestra que es factible su formación en un cátodo, asemejando el proceso de oxidación avanzada (AOP), conocido como electro-Fenton. La elevada reactividad del ${ }^{\bullet} \mathrm{OH}$ para llevar a cabo la destrucción de compuestos biorrefractarios y recalcitrantes está plenamente probada y justifica la propuesta de ensayar materiales nanoestructurados para multiplicar su producción en una celda electroquímica. La evaluación de cátodos de tipo multifuncional, conteniendo Pt y Pt-Pd, soportados sobre nanotubos de carbón multipared (MWCNT) se realizó por voltamperometría cíclica, para determinar la producción in-situ del radical ${ }^{\circ} \mathrm{OH}$. De esta manera, el desarrollo de un nuevo proceso de reducción electroquímica de tipo multifuncional $\left(\mathrm{O}_{2}\right.$ a $\mathrm{H}_{2} \mathrm{O}_{2}$ y $\mathrm{H}_{2} \mathrm{O}_{2}$ a $\left.{ }^{\bullet} \mathrm{OH}\right)$ tiene una potencial aplicación en celdas electroquímicas ya concebidas para mejorar la calidad de agua tratada.
\end{abstract}

PALABRAS CLAVE: radical hidroxilo, Pt/MWCNT, calidad de agua tratada, proceso electro-Fenton.

ABSTRACT: This paper presents an electrochemical strategy to evaluate nanostructured materials with high potential to be used as multifunctional cathodes. The goal is to increase the capability of an electrochemical process to degrade and eliminate recalcitrant organic compounds in treated water. The object under study is the production of hydroxyl radical $\left({ }^{\circ} \mathrm{OH}\right)$, also identified as a powerful oxidant (2.8 V vs. ENH), only overcome by Fluorine (3.03 V vs. ENH). This radical is commonly formed on the surface of catalytic anodes or by Fenton reactions; however, this paper shows that it is also possible to form the radical on a cathode, simulating the advanced oxidation mechanism known as electro-Fenton. The high reactivity of ${ }^{\circ} \mathrm{OH}$ to destroy persistent compounds is plentifully proved and justify the use of electrocatalytic nanostructured materials to multiply its effectiveness in an electrochemical cell. The evaluation of multifunctional

* Centro de Investigación y Desarrollo Tecnológico en Electroquímica, Unidad Tijuana, carretera libre Tijuana-Tecate km 26.5, esq. Blvd Nogales Parque Ind. El Florido CP 22444, Tijuana, B.C., México, Tel. (664) 6453278. Correspondencia: (moropeza@cideteq.mx). 
cathodes containing Pt y Pt-Pd, supported on raw multiwalled carbon nanotubes, MWCNT, was done by cyclic voltammetry to validate the in-situ formation of ${ }^{\circ} \mathrm{OH}$ radical. At this point, authors visualize the feasibility to develop a new electrochemical process using a multifunctional electrodes, with Fe species in the electrode surface and supported electrocatalysts, to transform $\mathrm{O}_{2}$ into $\mathrm{H}_{2} \mathrm{O}_{2}$ and $\mathrm{H}_{2} \mathrm{O}_{2}$ into ${ }^{\circ} \mathrm{OH}$, accordingly to improve the quality of treated water without extra chemicals.

KEYWORDS: hydroxyl radical, Pt/MWCNT, quality of treated water, electro-Fenton process.

\section{Introducción}

El radical hidroxilo $(\cdot \mathrm{OH})$ es un agente altamente oxidante, con potencial aplicación en electrosíntesis, industria farmacéutica, limpieza de superficies y tratamiento de aguas residuales. De acuerdo con lo anterior, la producción de esta especie y la mejora en los procesos que dan lugar a su formación es de creciente interés para la investigación en ciencia de materiales, fisicoquímica y medio ambiente. En el área de tratamiento de aguas residuales, el uso de procesos de oxidación avanzada (AOPs, advanced oxidation processes), que son procesos basados en la producción del ${ }^{\bullet} \mathrm{OH}$, ha demostrado superioridad en comparación al uso de procesos convencionales tales como ozonificación y coagulación (Ongen et al., 2012, Baig y Liechti, 2001), oxidación química y adsorción física (Ahn et al., 1999), y sistemas biológicos (Jothimani et al., 2003, Chengalroyen, 2012), para la destrucción de compuestos orgánicos de naturaleza recalcitrante. En general, los AOPs son preferidos sobre procesos convencionales por permitir la destrucción de contaminantes altamente tóxicos hasta $\mathrm{CO}_{2}$ y agua. Dentro de los AOPs, más representativos, se encuentran aquellos de tipo químico (fenton, fotofenton, ozonización en medio alcalino, $\mathrm{H}_{2} \mathrm{O}_{2} / \mathrm{UV}, \mathrm{O}_{3} / \mathrm{UV}, \mathrm{H}_{2} \mathrm{O}_{2} / \mathrm{O}_{3} / \mathrm{UV}$ ), fotocatálisis heterogénea, electroquímicos (electrofenton, fotoelectrofenton y oxidación electroquímica), haciendo uso de luz solar o asistida. Entre éstos, el proceso Fenton (Fenton, 1894), basado en la producción del $\bullet \mathrm{OH}$ (Ec. 1) es potencialmente aplicable en tratamiento de aguas residuales; sin embargo, parámetros como $\mathrm{pH}$, relación $\mathrm{Fe} / \mathrm{H}_{2} \mathrm{O}_{2}$, composición fisicoquímica y naturaleza de la muestra a tratar deben ser consideradas (Peralta et al. 2005).

$$
\mathrm{Fe}^{2+}+\mathrm{H}_{2} \mathrm{O}_{2} \longrightarrow \mathrm{Fe}^{3+}+\cdot \mathrm{OH}+\mathrm{OH}^{-} \quad \text { Ec. } 1
$$

De acuerdo con lo anterior, si el proceso no es debidamente optimizado, los ${ }^{\bullet} \mathrm{OHs}$ pueden reaccionar entre sí, dando lugar a especies con un potencial de oxidación menor, como el peróxido de hidrógeno, $\mathrm{H}_{2} \mathrm{O}_{2}$. El $\mathrm{H}_{2} \mathrm{O}_{2}$, también posee la capacidad de actuar como secuestrador del radical $\bullet \mathrm{OH}$ (Ec. 2), dando lugar a especies con menor potencial de oxidación como el radical perhidroxilo (Ec. 3). Si la dosis del ion $\mathrm{Fe}^{2+}$ no es la adecuada, puede reaccionar con el radical $\bullet \mathrm{OH}$, dando lugar a iones hidroxilo y $\mathrm{Fe}^{3^{+}}$(Ec. 4), afectando la eficiencia de la reacción. 


$$
\begin{array}{ll}
\cdot \mathrm{OH}+\cdot \mathrm{OH} \longrightarrow \mathrm{H}_{2} \mathrm{O}_{2} & \text { Ec. } 2 \\
\cdot \mathrm{OH}+\mathrm{H}_{2} \mathrm{O}_{2} \longrightarrow \mathrm{HO}_{2} \cdot \mathrm{H}_{2} \mathrm{O} & \text { Ec. } 3 \\
\mathrm{Fe}^{2^{+}}+\cdot \mathrm{OH} \longrightarrow \mathrm{Fe}^{3^{+}}+\mathrm{OH}^{-} & \text {Ec. } 4
\end{array}
$$

Aunque las reacciones (2-4) pueden ser controladas, la principal limitante del proceso vía química es el uso de $\mathrm{H}_{2} \mathrm{O}_{2}$ comercial, representando altos costos y el peligro latente de accidentes debido su transporte en altas cantidades. Considerando lo anterior, una mejora del proceso fue obtenida mediante el uso de la electroquímica, dando lugar al proceso Electro-Fenton, EF, en el cual el $\mathrm{H}_{2} \mathrm{O}_{2}$, necesario para llevar acabo la reacción Fenton (1) (Fenton y col., 1894), es generado electroquímicamente, a través del proceso de reducción de oxígeno vía 2 e $^{-}$(5).

$$
\mathrm{O}_{2}+2 \mathrm{H}^{+}+2 \mathrm{e}^{-} \longrightarrow \mathrm{H}_{2} \mathrm{O}_{2}
$$

A su vez, y a pesar de las mejoras en el proceso EF, la formación eventual de complejos (ácido oxálico- $\mathrm{Fe}^{3+}$ ) ocurre (Oturan y Brillas, 2007), y el uso de luz UV-A, de manera asistida, es aplicada, dando lugar al proceso conocido como FEF. A pesar de las ventajas que el uso de luz UV-A y la producción in situ del $\mathrm{H}_{2} \mathrm{O}_{2}$ ofrecen en un proceso EF, los retos actuales que enfrenta dicho proceso pueden agruparse en dos principales enfoques, i) uso de soportes del ion fierro, ii) síntesis de materiales con elevada producción $\mathrm{H}_{2} \mathrm{O}_{2}$. Con respecto al primer enfoque, el uso de membranas de nafion y resinas de intercambio iónico (Ramírez y et al., 2010) ha demostrado ser una alterativa; sin embargo, el costo de los materiales empleados pudiera ser una limitante para su escalamiento a nivel industrial. Con respecto al segundo enfoque, especial atención debe ser dirigida en la selección del material de cátodo, siendo los materiales de carbón de mayor importancia en comparación al uso de electrodo metálicos, debido a sus propiedades electro-catalíticas. Los materiales de carbón han sido ampliamente utilizados en distintas aplicaciones electroquímicas como electrodos en baterías y celdas de combustible (Arenas et al., 2014; Morales et al., 2010), debido a características como baja toxicidad, resistencia química, alta conductividad eléctrica y baja actividad catalítica para la descomposición de $\mathrm{H}_{2} \mathrm{O}_{2}$. Particularmente en sistemas de EF, materiales como el grafito (Forti et al., 2007), carbón vítreo reticulado, fibras de carbón activado (7,8), nanotubos de carbón (Zhihui et al., 2007), telas de carbón/grafito y electrodos compuestos de tela de carbón/PTFE (Chavez et al., 2010; Wang et al., 2010; Zhihui et al., 2007; Mahnoud et al., 2009) han sido evaluados como electrodos. A su vez, entre los principales requisitos que debe cumplir el material de cátodo en un sistema EF es poseer una excelente actividad electrocatalítica hacia la reducción de $\mathrm{O}_{2}$, para dar lugar a la formación de $\mathrm{H}_{2} \mathrm{O}_{2}$ (Ec. 5). De acuerdo con lo anterior, el uso de cátodos utilizando MWCNT representa una excelente opción (Forti et al., 2007; Chavez et al., 2010; Wang et al., 2010; Zhihui et al., 2007). A su vez, ha 
sido demostrado que el pH y el dopaje de los MWCNT con nanopartículas metálicas y bimetálicas como Pt-Pd aumentan su actividad catalítica favoreciendo la producción de $\mathrm{H}_{2} \mathrm{O}_{2}$ (Zhihui et al., 2007). Considerando los trabajos anteriores en esta comunicación presentamos la evaluación de cátodos de tipo multifuncional, conteniendo nanopartículas Pt y Pt-Pd, soportados sobre nanotubos de carbón (MWCNT) sin purificar (con Fe residual en la superficie) para favorecer la producción catódica in situ del ${ }^{\bullet} \mathrm{OH}$, eliminando de ésta manera el uso de soportes adicionales y disminuyendo los costos en la posible implementación de un prototipo de tipo EF, con potencial para la aplicación en el tratamiento electroquímico de agua residual tratada.

\section{Materiales y métodos}

\section{Reactivos}

Ácido sulfúrico $\left(\mathrm{H}_{2} \mathrm{SO}_{4}, 97.90 \%\right)$, cumarina $\left(\mathrm{C}_{9} \mathrm{H}_{6} \mathrm{O}_{2}, 99.99 \%\right)$, Nafion 117 (5\%), isopropanol $\left(\mathrm{C}_{3} \mathrm{H}_{8} \mathrm{O}, 99 \%\right)$ fueron obtenidos de Aldrich. Peróxido $\left(\mathrm{H}_{2} \mathrm{O}_{2}, 30 \%\right)$ fue obtenido de J. T. Baker. Todas las soluciones fueron preparadas con agua Milli Q, (resistividad, $r=>18 \mathrm{MW} \mathrm{cm}$ a $25^{\circ} \mathrm{C}$ ). Los MWCNTs fueron preparados por métodos previamente reportados (Alonso-Núñez et al., (2006)) con la salvedad de que no pasaron por la etapa de purificación para eliminar el Fe residual de su síntesis.

\section{Procedimiento experimental}

\section{Preparación del cátodo}

Los diferentes materiales utilizados como cátodo fueron preparados con tintas que contienen MWCNT con Fe superficial y modificados con nanopartículas de $\mathrm{Pt}$ y $\mathrm{Pt}-\mathrm{Pd}$. El área geométricamente activa de cada material evaluado fue de $0.125 \mathrm{~mm}^{2}$. Las tintas también contienen Nafión 117 al 5\% e Isopropanol. Toda la mezcla se somete una agitación ultrasónica con una sonda Sonics and Materials, VCX750, $20 \mathrm{kHz}$, previa a su uso como material de recubrimiento en una barra de grafito puro.

\section{Caracterización estructural y análisis elemental}

Los electrodos preparados fueron caracterizados mediante Microscopía Electrónica de Barrido (SEM, Scanning Electron Mycroscopy) empleando un equipo marca JEOL 5300 acoplado a una sonda de Dispersión de Rayos X (EDX, Energy-dispersive X-ray spectroscopy), marca EDAX para análisis elemental. 


\section{Caracterización electroquímica}

Los perfiles electroquímicos de cada material de cátodo fueron obtenidos mediante voltamperometría cíclica, empleando un potenciostato/galvanostato marca AUTOLAB controlado por programa NOVA. El análisis fue realizado utilizando una celda convencional de tres electrodos, utilizando como contra-electrodo $\mathrm{Pt}$ y $\mathrm{Ag} / \mathrm{AgCl}$ como electrodo de referencia. El análisis fue realizado en $\mathrm{H}_{2} \mathrm{SO}_{4} 0.5 \mathrm{M}$, aplicando una velocidad de barrido de $100 \mathrm{mV} / \mathrm{s}$. El rango de potencial evaluado fue fijado entre $-0.6 \mathrm{~V}$ y $1 \mathrm{~V}$. Antes de cada medición, el sistema fue desoxigenado, mediante el uso de $\mathrm{N}_{2}$.

\section{Resultados y discusión}

\section{Caracterización estructural y análisis elemental}

La figura 1 muestra la micrografía obtenida de los MWCNT en ausencia de nanopartículas de Pt o Pt-Pd (electrocatalizadores) y su respectivo análisis elemental, mediante EDX, mostrando claramente la presencia de Fe superficial, mismo que de aquí en adelante será identificado como Fe/MWCNT. Como antes se mencionó el Fe es un elemento indispensable para llevar acabo la producción del ${ }^{\circ} \mathrm{OH}$ y en este caso en particular asegura la expectativa de producirlo sobre un cátodo por su presencia.

La figura 2 muestra la micrografía y su respectivo análisis elemental, para el material de electrodo que contiene nanopartículas de Pt. El análisis EDX muestra la presencia tanto de Fe como de Pt, este material se denotará como Fe/MWCNT-Pt. La micrografía permite apreciar las siluetas de los MWCNT y algunas zonas brillantes atribuidas a la presencia de cúmulos de

FIGURA 1. SEM de los MWCNT sin purificar, en ausencia de electrocatalizadores.
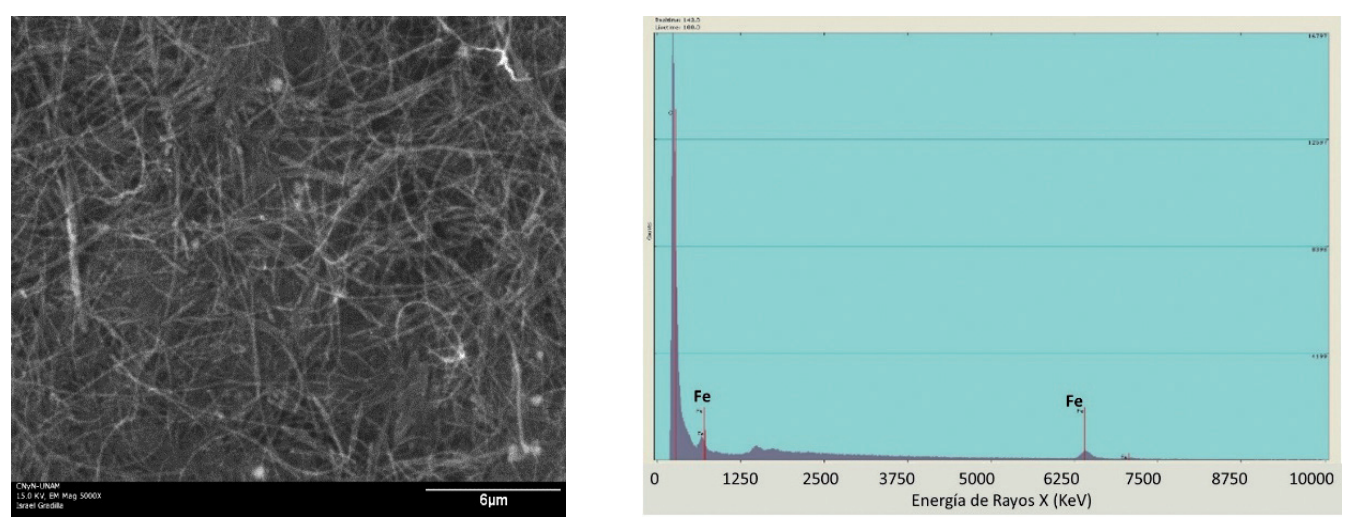

Fuente: Elaboración propia. 
FIGURA 2. SEM del material identificado como Fe/MWCNT-Pt.
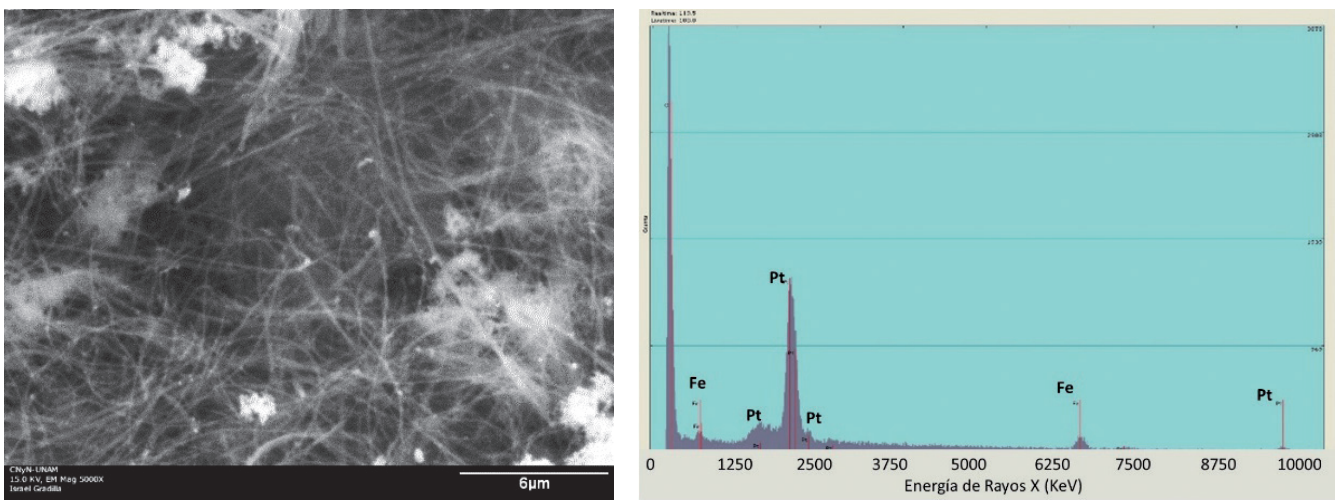

Fuente: Elaboración propia.

las nanopartículas de Pt, además de una especie de recubrimiento translucido sobre todos los MWCNT.

La figura 3 muestra las micrografías y el análisis elemental de los MWCNT con nanopartículas bimetálicas de Pt-Pd. De forma similar a la figura 2, se detectaron zonas brillantes atribuidas a la presencia de electrocatalizador bimetálico (Pt-Pd). A este material se le identificará ahora como $\mathrm{Fe} / \mathrm{MWCNT}-\mathrm{Pt}-\mathrm{Pd}$. En esta imagen SEM también se distingue un recubrimiento translucido que hace pensar en una alta densisdad de nanopartículas repartidas alrededor de todos los nanotubos.

El análisis SEM y EDX permitió confirmar claramente que el cátodo a

FIGURA 3. SEM del material identificado como Fe/MWCNT-Pt-Pd.
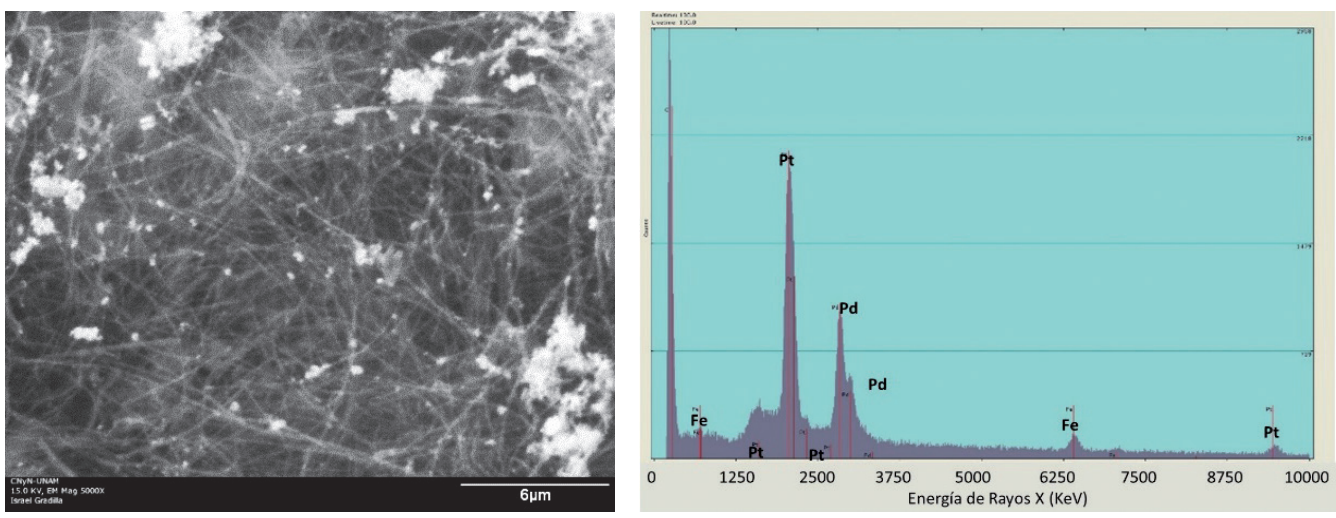

Fuente: Elaboración propia. 
evaluar contiene los materiales electrocatalizadores para la reacción de reducción de $\mathrm{O}_{2}$ (Pt, Pt-Pd) y el Fe para la formación de los radicales hidroxilos sobre su superficie. Si bien con esta caracterización no es factible distinguir el estado de oxidación del $\mathrm{Fe}$, se infiere que debe estar en forma de óxido por la exposición al ambiente y las condiciones de alta temperatura a las que es sometido durante la síntesis de los MWCNT.

\section{Caracterización electroquímica y análisis del radical hidroxilo}

Una vez realizada la caracterización morfológica y elemental de los diferentes materiales de cátodo a evaluar, se procedió a la caracterización electroquímica. La figura 4 muestra los perfiles voltamperometricos de cada electrodo preparado. En este análisis, las señales de oxidación/reducción de protones y oxígeno son distinguibles en los dos cátodos que contienen electrocatalizadores (Fe/MWCNT-Pt y Fe/MWCNT-Pt-Pd), así como la óxido/reducción de Fe superficial del electrodo denominado Fe/MWCNT (ver recuadro en la figura 4 ).

La respuesta electroquímica de cada material preparado en un electrolito como el $0.5 \mathrm{M}$ de $\mathrm{H}_{2} \mathrm{SO}_{4}$ es una prueba de calidad ya que se valida la electroactividad de los materiales preparados en ausencia de oxígeno y se puede distinguir la exposición de diferentes planos cristalinos en los materiales electrocatalizadores por el número de señales óxido/reducción atribuibles a los protones (región donde $\mathrm{E} \in(0,-200) \mathrm{mV}$ ). De esta manera, se cuenta con la evidencia de que los MWCNT son adecuados para soportar electrocatalizadores activos para la adsorción y reducción de protones, con su consecuente oxidación al invertir el barrido (región donde $\mathrm{E} \in(0,-200) \mathrm{mV}$ ), y la

FIGURA 4. Voltamperometría cíclica en $\mathrm{H}_{2} \mathrm{SO}_{4} 0.5 \mathrm{M}$ a una velocidad de barrido de $100 \mathrm{mV} / \mathrm{s}$, utilizando un electrodo de referencia de $\mathrm{Ag} / \mathrm{AgCl}$ y contraelectrodo de Pt, en celdas separadas, burbujeo constante con $\mathrm{N}_{2}$.

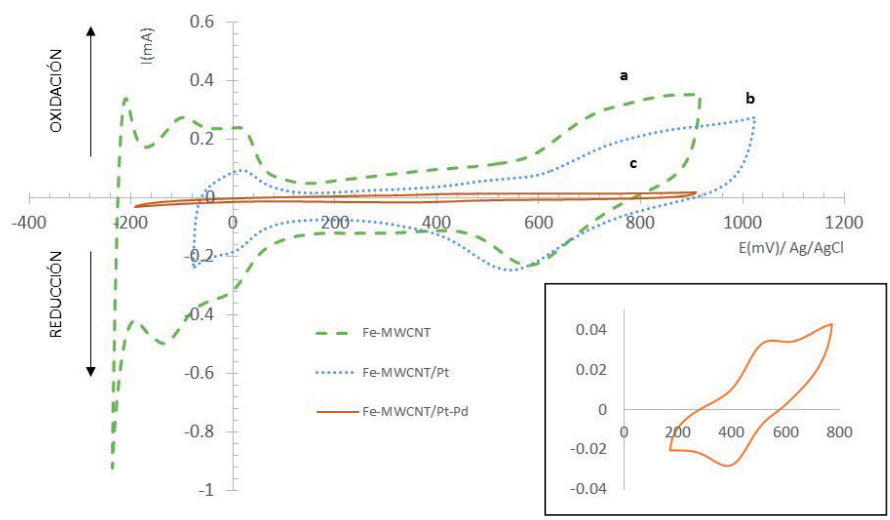

Fuente: Elaboración propia. 
FIGURA 5. Voltamperometría cíclica en $0.5 \mathrm{M} \mathrm{H}_{2} \mathrm{SO}_{4}$ a una velocidad de barrido de $100 \mathrm{mV} / \mathrm{s}$, utilizando un electrodo de referencia de $\mathrm{Ag} / \mathrm{AgCl}$ y contraelectrodo de Pt, en celda con división, burbujeo constante con $\mathrm{O}_{2}$.

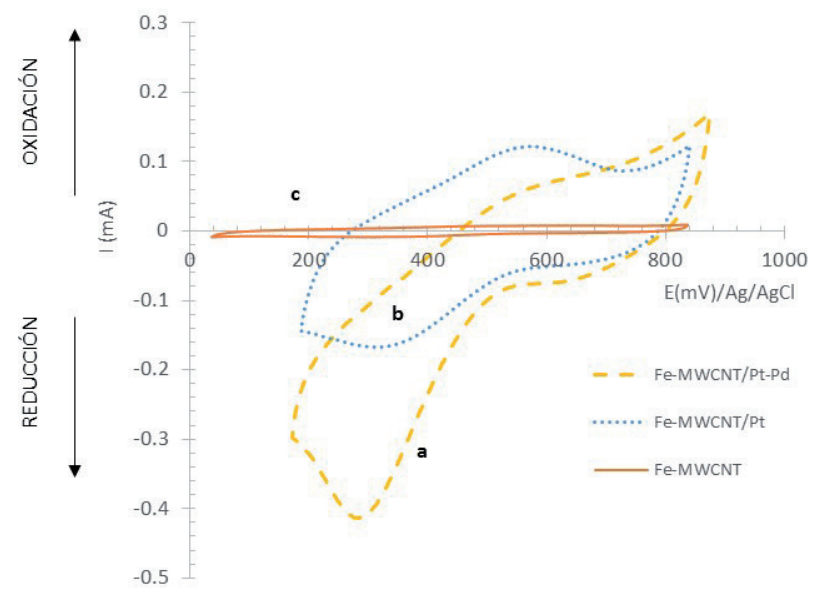

Fuente: Elaboración propia.

adsorción y oxidación de oxígeno con su consecuente reducción y formación de $\mathrm{H}_{2} \mathrm{O}_{2}$ en la superficie (región donde $\mathrm{E} \in(400,900) \mathrm{mV}$ ). El gráfico (a) corresponde al electrodo Fe/MWCNT-Pt-Pd, observando la señal de óxido-reducción del Pt entre 600 y 800 mV, además, en potenciales de reducción alrededor de $-100 \mathrm{mV}$ fueron observadas señales redox atribuidas a la presencia del Pd. El gráfico (b) es el electrodo de Fe/MWCNT-Pt y se observa que a $800 \mathrm{mV}$ se presenta la formación de un óxido de Pt y a $600 \mathrm{mV}$ su reducción, en la zona de reducción de protones no se manifiesta la exposición de diferentes planos cristalinos como en el caso de la curva (a). El gráfico (c) corresponde al electrodo Fe/MWCNT, que comparado con los que contienen catalizadores nanoparticulados no tiene electroactividad. Por otra parte, en el análisis comparativo (figura 4), las señales de óxido-reducción del hierro no alcanzan a apreciarse debido a la alta actividad catalítica de los electrodos modificados con MWCNTs-Pt y Pt-Pd. Sin embargo, los potenciales redox del Fe pueden ser claramente apreciados en un rango de 400 y $500 \mathrm{mV}$ (recuadro de la figura 4). Con el fin de identificar los potenciales de reducción de $\mathrm{O}_{2}$ en cada material de cátodo, y proceder a la evaluación de la producción de $\mathrm{H}_{2} \mathrm{O}_{2}$, se trazaron curvas de voltamperometría cíclica en medio ácido y presencia de $\mathrm{O}_{2}$. La figura 5 muestra el análisis comparativo de los diferentes perfiles voltamperométricos de cada material evaluado en el mismo electrolito de $0.5 \mathrm{M}$ de $\mathrm{H}_{2} \mathrm{SO}_{4}$, pero esta vez bajo una atmósfera saturada de oxígeno, para contar con el reactivo principal de la producción de $\mathrm{H}_{2} \mathrm{O}_{2}$.

Se observa que en presencia de Pt-Pd (Fe/MWCNT-Pt-Pd) la reducción de oxígeno es altamente catalizada, decreciendo dicha actividad, para los electrodos de Fe/MWCNT-Pt y totalmente nulo para Fe/MWCNT. Este 
análisis comprueba que las propiedades catalíticas de las partículas de Pt-Pd favorecen la reducción de $\mathrm{O}_{2} \mathrm{y}$, por lo tanto, la subsecuente formación de $\mathrm{H}_{2} \mathrm{O}_{2}$, lo cual fue evidenciado mediante UV-vis mediante el uso de oxisulfato de titanio (Brillas et al. 2009). De acuerdo con lo anterior, una mayor generación de $\mathrm{H}_{2} \mathrm{O}_{2}$, con sitios de Fe contiguos significaría una mayor producción del $\bullet \mathrm{OH}$, lo cual también fue verificado mediante espectroscopía de UV-vis y fluorescencia. La proyección de esta nueva forma de producir radicales ${ }^{\bullet} \mathrm{OH}$ en un cátodo tiene gran potencial de aplicación en el área ambiental y de la electrocatálisis, por lo que el grupo de investigación continua realizando pruebas de desempeño ya con agua tratada que contiene contaminantes recalcitrantes y difíciles de eliminar.

\section{Conclusiones}

Los resultados de la evaluación electroquímica del desempeño de cátodos multifuncionales para producir radicales ${ }^{\bullet} \mathrm{OH}$ dieron resultados prometedores, dado que la presencia de ellos fue corroborada por espectoscopías de UV-vis y espectroscopía de fluorescencia de cumanina. Es así que se pone de manifiesto que los cátodos diseñados con materiales nanoestructurados, Fe/MWCNT-Pt y Fe/MWCNT-Pt-Pd, son multifuncionales ya que pueden conjuntar las condiciones para formar los radicales ${ }^{\bullet} \mathrm{OH}$ como se hace en procesos electrofenton mediante la reducción electroquímica de $\mathrm{O}_{2}$ a $\mathrm{H}_{2} \mathrm{O}_{2}$ y de $\mathrm{H}_{2} \mathrm{O}_{2}$ a $\cdot \mathrm{OH}$ en presencia de Fe superficial. Estos materiales demuestran tener potencial aplicación en el tratamiento de agua residual y específicamente en el agua residual tratada, para aumentar su calidad.

\section{Referencias}

A. Aguilar-Elguézabal, W. Antúnez, G. Alonso-Núñez, F. Paraguay Delgado, F. Espinoza, M. Miki-Yoshida. (2006). Study of carbon nanotubes synthesis by spray pyrolysis and model of growth. Diamond and Related Materials, vol. 15: 1329.

A. M. Oturan, E. Brillas (2007). Electrochemical advanced oxidation processes (EAOPs) for environmental applications. Portugaliae Electrochimica Acta, vol. 25: 1.

A. Ongen. Y. Gunes, I. Talinli (2012). Treatability of pesticide industry effluent by ozonation and coagulation. A real effluent investigation. Journal of Environmental Protection and Ecology, vol. 13: 23.

A. Zhihui, T. Mei, J. Liu, J. Li, F. Jia, L. Zhang, J. Qiu (2007). Fe@Fe $\mathrm{O}_{3}$ Core-Shell nanowires as an iron reagent. Their combination with CNTs as an effective oxygen-fed gas diffusion electrode in a neutral electro-Fenton system. The Journal of Physical Chemistry, vol. 111, núm. 40: 14799.

D. H. Ahn., W. S. Chang, T. I. Yoon (1999). Dyestuff wastewater treatment using chemical oxidation, physical adsorption and fixed bed biofilm process. Process Biochemistry, 34: 429-439. 
D. Morales-Acosta, J. Ledesma-García, L. A. Godínez, H. G. Rodríguez (2010). Development of $\mathrm{Pd}$ and $\mathrm{Pd}-\mathrm{Co}$ catalysts supported on multi-walled carbon nanotubes for formic acid oxidation. Journal of Power Sources, vol. 195, núm. 2: 461.

E. Brillas, I. Sirés, M. A. Oturan (2009). Electro-Fenton process and related electrochemical technologies based on Fenton's reaction chemistry. Chemical Reviews, vol. 109: 6670.

E. Isarain-Chavez, C. Arias, P.L. Cabot, F. Centellas, R. M. Rodríguez, J. A. Garrido, E. Brillas (2010). Mineralization of the drug $\beta$-blocker atenolol by electro-Fenton and photoelectro-Fenton using an air-diffusion cathode for $\mathrm{H}_{2} \mathrm{O}_{2}$ electrogeneration combined with a carbon-felt cathode for $\mathrm{Fe}^{2+}$ regeneration. Applied Catalysis, B: Environmental, vol. 96, núm. 3-4: 361.

H. J. H. Fenton (1894). Oxidation of tartaric acid in the presence of iron. Journal of chemical society, vol. 65: 899.

I. Muñoz, S. Malato, A. Rodríguez, X. Domènech (2008). Integration of environmental and economic performance of processes. Case study on advanced oxidation processes for wastewater treatment. Journal of Advanced Oxidation Technologies, vol. 11, núm. 2: 270.

J. C. Forti, R. S. Rocha, M. R. V. Lanza, R. Bertazzoli (2007). Electrochemical synthesis of hydrogen peroxide on oxygen-fed graphite/PTFE electrodes modified by 2-ethylanthraquinone. Journal of Electroanalytical Chemistry, vol. 601, núm. 1: 63.

J. M. Peralta, S. Mejía, L. A. Godínez, Y. Meas (2005). Applications of analytical chemistry in environmental research. Fenton an electrochemical approaches for water purification technologies. Research Signpost. Trivandrum, Kerala, India, 101.

J. Ramírez, L. A. Godínez, M. Méndez, Y. Meas, F. J. Rodríguez (2010). Heterogeneous photo-electro-Fenton process using different iron supporting materials. Journal Applied. Electrochemistry, vol. 40: 1729.

M. D. Chengalroyen, E. R Dabbs (2012). The microbial degradation of azo dyes: minireview. World Journal of Microbiology and Biotechnology, vol. 29, núm. 3, 389.

P. Jothimani., G. Kalaiselvan, A. Baskaran, S. D. Augustine, K. Ramaswamy (2003). Anaerobic biodegradation of aromatic compounds. Indian Journal of Experimental Biology, vol. 141: 1046.

R. Arenas-Carmona, D. Morales-Acosta, A. Martinez-Luevanos, F.J. Rodríguez-Varela (2014). Comparative study of the electrocatalytic oxidation of glycerol on $\mathrm{Pd}-\mathrm{Au} / \mathrm{CMO}$ and $\mathrm{Pd}-\mathrm{Au} / \mathrm{MWCNT}$ nanocatalysts prepared by the polyol method in alkaline media. ECS Transactions, vol. 64, núm. 3: 1061.

R. G. Zepp, B. C. Faust, J. Hoigne (1992). Hydroxyl radical formation in aqueous reactions ( $\mathrm{pH} 3-8$ ) of iron (II) with hydrogen peroxide: the photo-Fenton reaction. Environmental Science Technology, vol. 26: 6670.

S. Baig, P. A. Liechti (2001). Ozone treatment for bio-refractory COD removal. Water Science and Technology, vol. 43, núm. 2: 97. 
Mundo Nano | Artículos | vol. 8, No. 14, enero-junio, 2015 | www.mundonano.unam.mx

W. Chih-Ta, C. Wei-Lug, C. Mei-Hui, K. Yi-Ming (2010). COD removal from real dyeing wastewater by electro-Fenton technology using an activated carbon fiber cathode. Desalination, vol. 253. núm. 1-3: 129.

Z. Mahnoud, D. Salari, A. Niaei, A. Khataee (2009). Peroxi-coagulation degradation of C.I. Basic yellow 2 based on carbon-PTFE and carbon nanotube-PTFE electrodes as cathode. Electrochimica Acta, vol. 54, núm. 1-2: 6651. 